\title{
Noninvasive spinal neuromodulation to map and augment lower urinary tract function in rhesus macaques
}

\author{
Leif A. Havton a, b, *, Kari L. Christe c , V. Reggie Edgerton b, d, e, f, g, h, Parag N. Gad ${ }^{\text {d }}$ \\ a Department of Neurology, David Geffen School of Medicine at University of California, Los Angeles, Los Angeles, CA, USA \\ b Department of Neurobiology, David Geffen School of Medicine at University of California, Los Angeles, CA, USA \\ ${ }^{c}$ California National Primate Research Center, University of California, Davis, Davis, CA, USA \\ d Department of Integrative Biology and Physiology, University of California, Los Angeles, Los Angeles, CA, USA \\ e Department of Neurosurgery, David Geffen School of Medicine at University of California, Los Angeles, CA, USA \\ ${ }^{\mathrm{f}}$ Brain Research Institute, University of California, Los Angeles, Los Angeles, CA 90095, USA \\ ${ }^{\mathrm{g}}$ Institut Guttmann, Hospital de Neurorehabilitació, Institut Universitari adscrit a la Universitat Autònoma de Barcelona, Badalona 08916, Barcelona, Spain \\ ${ }^{\mathrm{h}}$ Centre for Neuroscience and Regenerative Medicine, University of Technology, Sydney, NSW, Australia
}

\section{ART ICLE INFO}

\section{Keywords:}

Non-human primate

Cystometrogram

Electromyography

Transcutaneous spinal cord stimulation

\begin{abstract}
A B S T R A C T
Dysfunction of the lower urinary tract (LUT) is prevalent in neurological disorders, including multiple sclerosis, stroke, spinal cord injury and neurodegenerative conditions. Common symptoms include urgency, incontinence, and urinary retention. Recent advances in neuromodulation have resulted in improved treatments for overactive bladder symptoms of urgency, frequency, and nocturia. However, there are presently no treatments available for the induction of voiding to overcome urinary retention. We demonstrate that transcutaneous spinal cord stimulation (TSCS), a non-invasive intervention, applied over the thoracolumbar spine in neurologically intact rhesus macaques can activate the LUT, including activation of the bladder detrusor muscle, the urethral sphincter and pelvic floor muscles. Urodynamic studies show improved voiding efficiency and decreased post-voiding residual volumes in the bladder, while maintaining coordinated activity in the detrusor and sphincter with physiologic detrusor peak pressure, contraction duration, and urine flow rate remaining unchanged. We conclude that TSCS may represent a novel approach to activate the LUT and enable voiding in select neurological conditions.
\end{abstract}

\section{Introduction}

A variety of electrical stimulation strategies are known to modulate lower urinary tract function (LUT) in experimental models and humans. For instance, electrical stimulation of sacral nerve roots, pudendal nerve, or tibial nerve may modulate micturition reflexes, suppress urgency, and augment LUT function in patients with an overactive bladder (Bartley et al., 2013; Brindley et al., 1982; Lordêlo et al., 2010; Peters et al., 2010; Staskin et al., 2012).

Epidural spinal stimulation may also be used to augment LUT function in neurologically intact rats (Abud et al., 2015) and after a spinal cord injury in rats (Abud et al., 2015; Gad et al., 2014). Modulation of LUT function with improved functional outcomes were also demonstrated when epidural stimulation was combined with locomotor train- ing after SCI in rats (Horst et al., 2013) and humans (Hubscher et al., 2018).

However, the above stimulation procedures are invasive or may bypass the normal automaticity of spinal sensorimotor circuits. Interestingly, transcutaneous electrical spinal cord stimulation (TSCS) has emerged as a non-invasive strategy to activate neural networks in the lumbosacral spinal cord and generate coordinated stepping in neurologically intact human subjects and after severe spinal cord injury (SCI) (Gerasimenko et al., 2015a; Gerasimenko et al., 2015b). However, the use of TSCS for the activation of micturition reflexes and to initiate voiding has not been explored previously. In the present study, we have investigated the potential utility of TSCS for neuro-urological applications. The studies were performed in adult rhesus macaques, a clinically relevant and translational research model for studies of pelvic organ physiology, reflex micturition and LUT function (Chang and Havton, 2016; Gad et al., 2017; Gad et al., 2018a, 2018b, 2018c).

\footnotetext{
* Corresponding author at: Departments of Neurology and Neurobiology, David Geffen School of Medicine at UCLA, 710 Westwood Plaza, Los Angeles, CA 90095, USA.

Email address: lhavton@mednet.ucla.edu (L.A. Havton)
} 
The present study addressed three principal aims. First, feasibility of using TSCS to activate micturition reflexes and to enable voiding was evaluated in neurologically intact rhesus macaques using urodynamic evaluations. Second, the effects of varied stimulus location and strength over the thoracolumbar spine on evoked responses from the detrusor and external urethral sphincter were determined. Third, the functional characteristics of TSCS-enabled voiding were compared to the corresponding properties of physiologic reflex micturition in the same subjects.

\section{Methods}

A total of 9 female rhesus macaques (Macaca mulatta) were included in the study. All subjects were adults aged $19.41 \pm 2.52$, weighed $9.04 \pm 0.70 \mathrm{~kg}$ and were housed at the California National Primate Research Center (CNPRC), all procedures were performed in compliance with the Guide for the Care and Use of Laboratory Animals (2011) and approved by the UC Davis Institutional Animal Care and Use Committee (IACUC). Each subject was immobilized by an intramuscular (IM) dose of ketamine $(10 \mathrm{mg} / \mathrm{kg}$ ) followed by placement of an IV catheter and an endotracheal tube for airway protection. Ketamine was next administered by constant rate infusion, initially at $10-12 \mathrm{mg} / \mathrm{kg} / \mathrm{h} \mathrm{IV}$, and the dose was individually adjusted for sedation and immobilization.

\subsection{Catheter and EMG electrode placement}

The perineal area was prepared for an aseptic transurethral catheter placement using three alternating applications of a $10 \%$ povidone-iodine solution and 70\% isopropyl alcohol. A 7-Fr triple lumen transurethral bladder catheter (Life-Tech) was lubricated and placed with the distal end positioned within the bladder and the urethral port within the urethral canal. The proximal ends of the cystometry port and the urethral pressure profile (UPP) port were individually attached to a TSD 104A pressure transducer and connected to an MP 150 Data Acquisition System (Biopac), which in turn was connected to a personal computer equipped with the AcqKnowledge software (Biopac). Both pressure channels were calibrated using a two point calibration system. Bilateral pairs of wire-electrodes (1215A-F, Life-Tech) were inserted into the external urethral sphincter using surface landmarks. Paired 22 gauge needle electrodes (Hamilton Company, Reno, NV) were placed into the external anal sphincter and surface patch electrodes were attached to the pelvic floor, laterally to the external anal sphincter and over the levator ani muscle.

\subsection{Noninvasive spinal cord stimulation protocol}

Transcutaneous Spinal Cord Stimulation (TSCS) was delivered along the midline between spinous processes at T11-T12, T12-L1, L1-L2, L2-L3 and L3-L4 to map the multiple levels. The intensity of stimulation at each level was set at $100 \mathrm{~V}$ with pulse width varying from $1 \mathrm{~ms}$ to $5 \mathrm{~ms}$ in steps of $1 \mathrm{~ms}$. Electrical stimulation was delivered between spinous processes using $2.0 \mathrm{~cm}$-diameter round gel adhesive electrodes (Axelgaard, ValuTrode ${ }^{\circledR}$ Cloth) as cathode and a $5.0 \times 10.0 \mathrm{~cm}^{2}$ rectangular electrodes (Axelgaard, ValuTrode ${ }^{\circledR}$ Cloth) placed in the midline and attached to the skin over the belly as anode. The stimulation was issued using monophasic rectangular pulses at a frequency of $1 \mathrm{~Hz}$. Each intensity of stimulation determined by the pulse width was repeated 5 times to assess reproducibility of responses. Throughout the TSCS protocol, bladder and urethral pressures as well as EUS, EAS and Pelvic floor EMG activity were recorded.

\subsection{Urodynamic studies}

For urodynamic studies, the bladder was first emptied using a syringe connected to the catheter fill port. To evoke a micturition reflex, the bladder was manually infused with saline using a syringe attached to the fill port of the catheter. The rate of filling was consistently maintained at $60 \mathrm{ml} / \mathrm{min}$. The bladder pressure was continually monitored, and infusion was performed until a bladder baseline pressure, typically $0-5 \mathrm{~cm} \mathrm{H}_{2} \mathrm{O}$, had increased to $20 \mathrm{~cm} \mathrm{H}_{2} \mathrm{O}$. A reflex micturition response was typically observed within 30-60 s with a delayed increase in bladder pressure and start of voiding when the voiding threshold had been reached. Start of voiding was marked when saline flow from the urethra was observed. End of voiding was marked when flow ceased. Voided saline was collected in a cup to measure the voided volume. Reflex micturition was documented by urodynamic and EMG recordings.

\subsection{Urodynamic studies with TSCS}

The inter-spinous process level that generated the greatest change in detrusor pressure was selected for micturition reflex studies with TSCS. The bladder was emptied using a syringe connected to the catheter fill port. The bladder was infused manually with saline using a syringe attached to the fill port of the catheter. The rate of filling was consistently maintained at $60 \mathrm{ml} / \mathrm{min}$. The bladder pressure was continually monitored and infusion was performed until the bladder pressure had increased to a sub-threshold level of $15-18 \mathrm{~cm} \mathrm{H}_{2} 0$. After completion of the bladder infusion, the bladder pressure was monitored for at least 2 to $3 \mathrm{~min}$ to ensure for the absence of reflex micturition. Next, TSCS was administered for 60s at a frequency of $1 \mathrm{~Hz}, 100 \mathrm{~V}$ intensity and pulse width of $5 \mathrm{~ms}$. Start of voiding was marked when saline flow from the urethra was observed. End of voiding was marked when flow ceased. Voided saline was collected in a cup to measure the voided volume. TSCS enabled voiding was documented using urodynamic and EMG recordings.

\subsection{Data and statistical analysis}

The studies provided several outcome measures including: 1) infused volume; 2) voided volume; 3) post voiding residual; 4) voiding efficiency (voided volume/infused volume); 5) voiding flow rate (voided volume/voiding duration); 6) peak pressure during detrusor contraction; 7) contraction duration; 8) peak-to-peak pressures and EMG amplitude during mapping studies; 9) delay between completion of filling and onset of voiding; 10) delay between onset of TSCS and onset of voiding. All data were reported as mean \pm SE. The non-parametric Mann Whitney $U$ test was used to statistically compare data between groups TSCS Off and TSCS On using Graphpad software. The criterion level for the determination of a statistical difference was set at $P<.05$ for all comparisons.

\section{Results}

The present study evaluated the potential feasibility for TSCS to modulate micturition reflexes and enable voiding in neurologically intact rhesus macaques. For this purpose, TSCS was first performed in combination with comprehensive urodynamic studies to map evoked responses from the lower urinary tract. TSCS was applied along the midline at $5(n=7)$ or $8(n=1)$ consecutive inter-spinous process locations of the thoracolumbar spine at different stimulation durations. The evoked responses were recorded as bladder pressure, urethral pressure, and EUS EMG recordings (Fig. 1). Due to the mechanical nature of contractions recorded for the detrusor and urethral pressure and electro- 




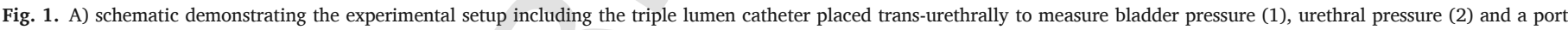

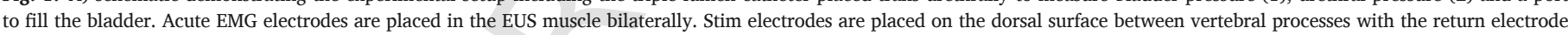

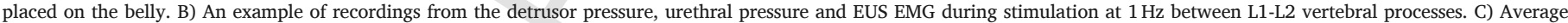

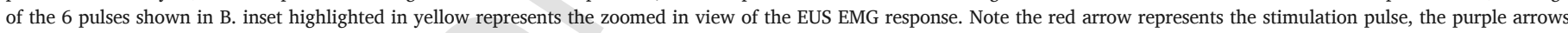

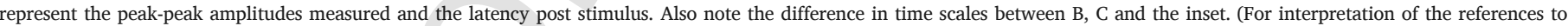
colour in this figure legend, the reader is referred to the web version of this article.)

physiological response picked up by the EUS EMG electrodes, the time course of response varied significantly. The pressure response was observed in the 100 to $300 \mathrm{~ms}$ window, whereas the electrophysiological response was recorded in the $6-20 \mathrm{~ms}$ window (Fig. 1C). The peak pressure in the detrusor and urethra was observed at $\sim 100 \mathrm{~ms}$, with the peak EMG in the EUS at $\sim 8 \mathrm{~ms}$ post stimulation pulse. Next, functional maps were generated from each subject for bladder pressure, urethral pressure and EUS EMG activity to display optimal inter-spinous process location and stim duration for the evoked responses (Fig. 2). In each of the subjects, specific locations could be identified that demonstrated high pressure detrusor pressure change and high EUS EMG response ( $\sim \mathrm{L} 1$ in subject 1 and 2 and $\sim \mathrm{L} 3$ in subject 3$)$. Increasing the intensity of stimulation by changing the pulse width resulted in increased responses in detrusor pressure, urethral pressure and EUS EMG. Distinct hot spots were observed in the heat maps (Fig. 2B) while changing the pulse-width and site of stimulation with specific over- laps between the detrusor, urethral pressure and EUS EMG. However, the EUS EMG responses were observed down till the L3 level, with little to no urethral pressure response.

Urodynamics studies (UDS) were performed in five subjects first without stimulation followed by with TSCS. While performing UDS without stimulation, saline was filled in via the triple lumen catheter till the pressure in the detrusor reached $\sim 20 \mathrm{~cm}$ of $\mathrm{H}_{2} \mathrm{O}$. This increased pressure resulted in physiological contraction leading to voiding (Fig. 3A, black trace). Next, the bladder was partially filled with saline to a pressure of 15- $18 \mathrm{~cm} \mathrm{H}_{2} \mathrm{O}$, a volume below the threshold for evoking a reflex bladder contraction in rhesus macaques under light ketamine sedation. The bladder pressure was monitored for 2-3 min to ensure a reflex contraction wasn't initiated. TSCS was initiated for a period of $60 \mathrm{~s}$ resulting in an evoked detrusor contraction and voiding. The TSCS was administered at the inter-spinous process interspace for L1/L2 ( $n=$ 


\section{A} Variation with Site of Stimulation
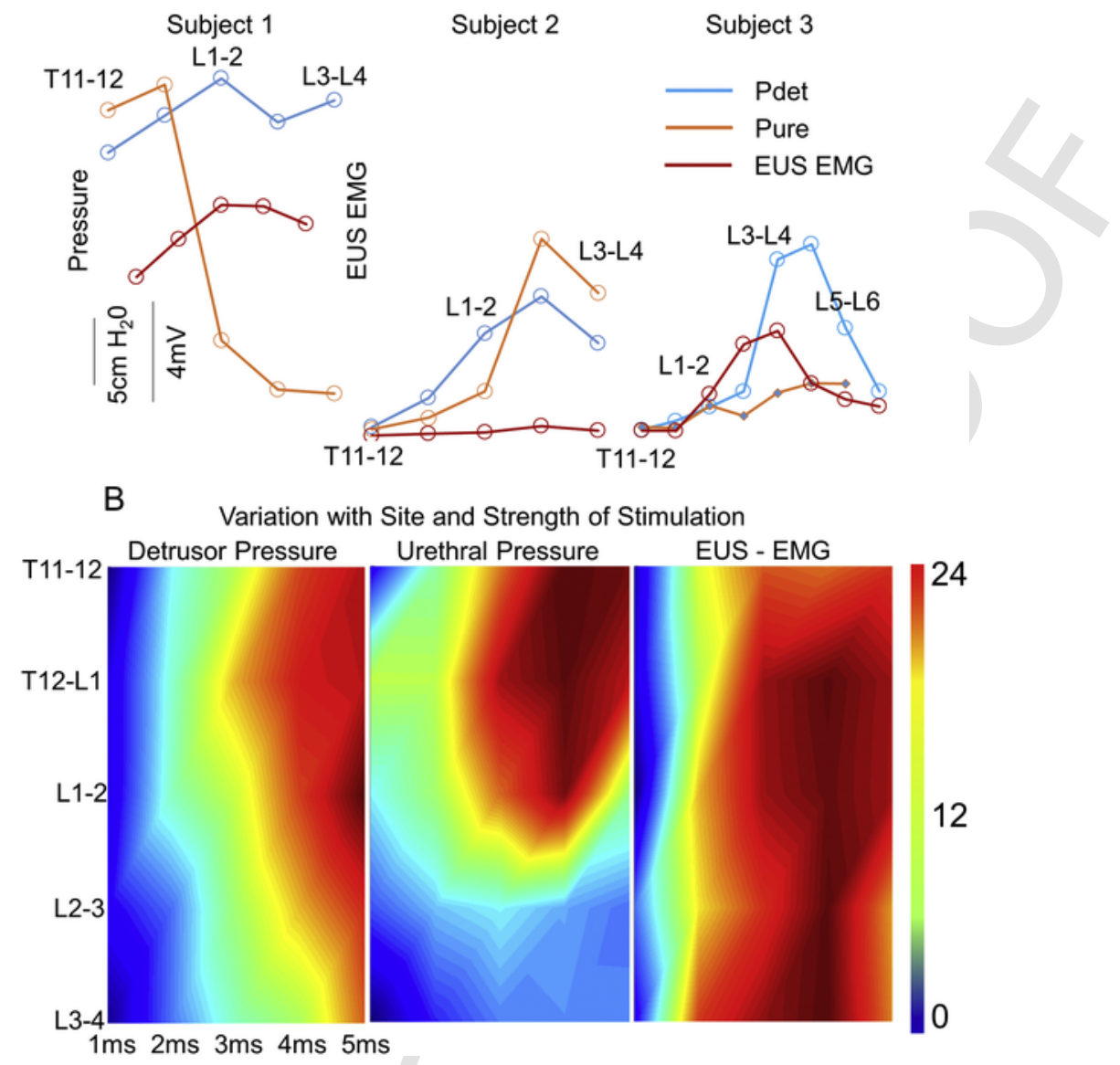

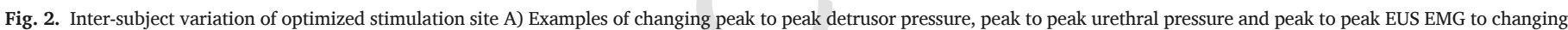

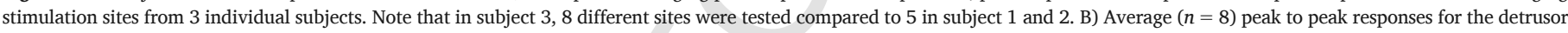
pressure, urethral pressure and EUS EMG to changing site and pulse width of stimulation. The colour represents the amplitude based on the adjacent heat map.

3) or L3/L4 $(n=2)$ based on prior functional maps obtained from each subject.

TSCS resulted in a significantly improved VE of $32.8 \pm 10.4 \%$ and decreased PVR of $67.6 \pm 9.8 \%$ compared to corresponding VE of $18.2 \pm 8.8 \%$ and PVR of $81.4 \pm 7.5 \%$ associated with reflex micturition in the same subjects $(n=5)$ (Figs. 3,4$)$. There was no difference between voiding flow rate, duration of the voiding contraction, and peak pressure during voiding between TSCS-enabled voiding and physiologic reflex micturition. The time between start of TSCS and onset of voiding in animals with sub-threshold bladder filling was $73 \pm 25 \mathrm{~s}(\mathrm{n}=5)$ and not statistically different from the delay of $50 \pm 19 s(n=5)$ after the completed filling of the bladder the bladder to a threshold pressure of $20-25 \mathrm{~cm} \mathrm{H}_{2} \mathrm{O}$ and the onset of reflex voiding. In some animals, the TSCS-evoked voiding during the period of active electrical stimulation, whereas voiding onset took place after the completion of the electrical stimulation in other subjects (Fig. 3). In all the nine cases tested, the subjects tolerated the stimulation bouts, both mapping and UDS, with no damage to at the stimulation sites. All animals made full recovery from the sedation and procedure at the end of each experiment.

\section{Discussion}

The present studies demonstrated feasibility for TSCS to activate micturition reflexes and enable voiding in adult rhesus macaques. The studies provide support for the notion of a non-invasive approach to activate the spinal cord circuitry to modulate LUT function. Our findings support the concept of using electrical stimulation of the spinal cord to activate neural networks for eliciting and modulating micturition reflexes.

Earlier studies have shown feasibility of using epidural spinal stimulation to selectively activate neural networks in experimental models. Epidural stimulation of the L3 segment in rats activated the spinal EUS controlling center and promoted the switch from bladder storage to voiding in neurologically intact subjects and after a spinal cord injury (Abud et al., 2015) or a pelvic nerve crush injury (Chang et al., 2018). Epidural stimulation that enables locomotor activity in rats may also influence neural networks controlling LUT function and initiate bladder emptying in rats with an SCI (Gad et al., 2014). These studies highlight the automaticity that is intrinsic to the spinal networks controlling LUT function and the ability of using epidural stimulation to activate the appropriate neural networks based on the site and pattern of stimulation. In a recent study on functional mapping of the LUT in rhesus macaques, we have demonstrated a similar site and stimulation dependent activation of spinal cord networks using TSCS as a non-invasive approach (Gad et al., 2018a). However, our recent study on functional mapping in non-human primates studied responses to TSCS using an empty bladder and did not attempt to induce voiding (Gad et al., 2018a). Collectively, these epidural stimulation and TSCS studies suggest activation of the spinal networks controlling LUT function with coordinated detrusor and urethral sphincter activation to enable efficient voiding with minimal detrusor-sphincter-dyssynergia in both neurologically intact subjects and after an SCI. The absence of a significant difference in the delay between the completion of filling and initiation of voiding and the TSCS onset and the start of voiding suggests a simi- 


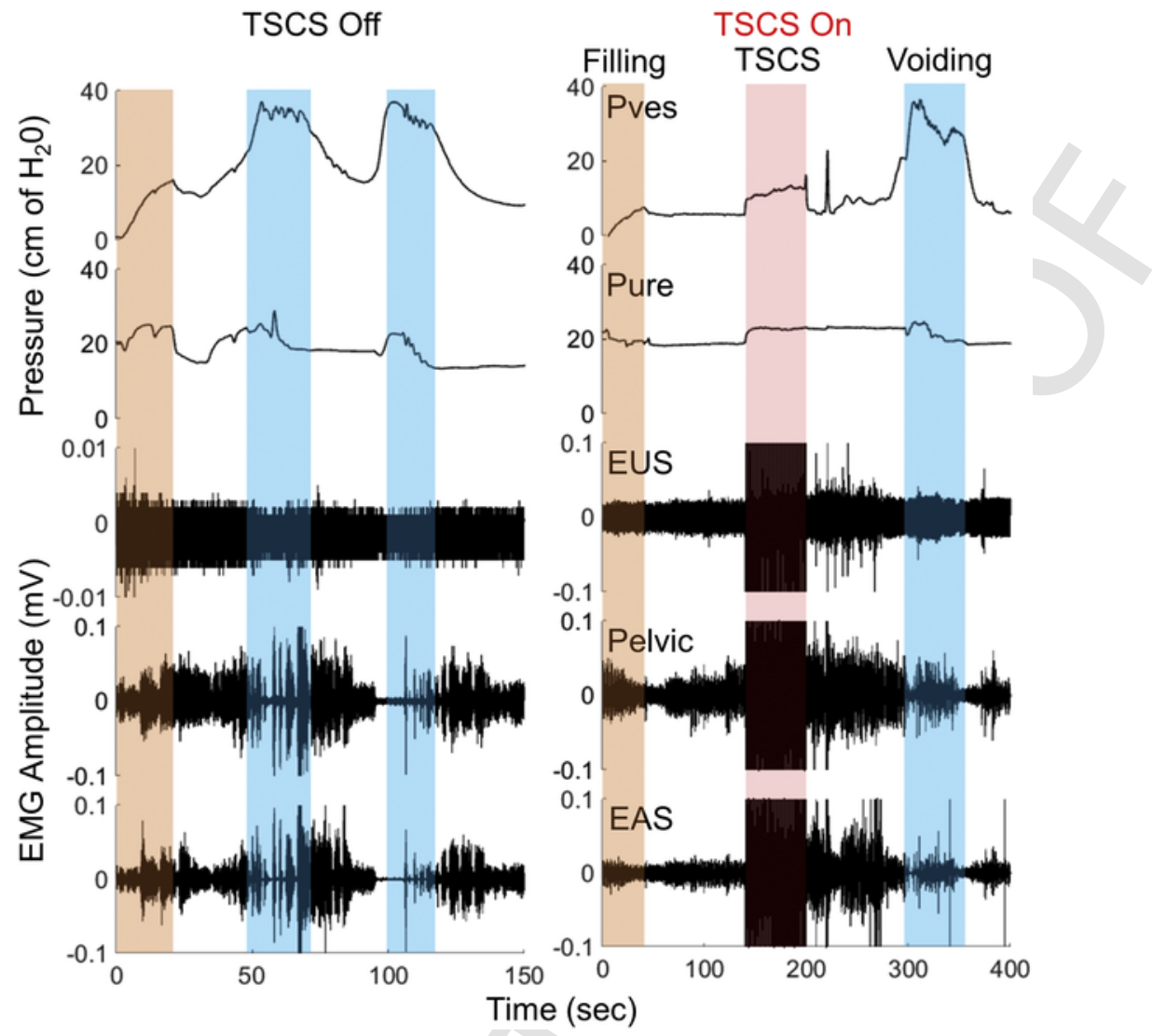



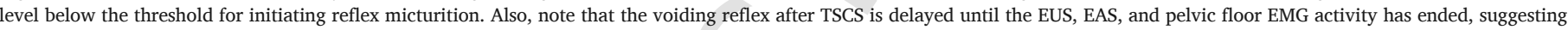



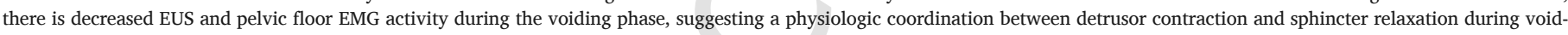

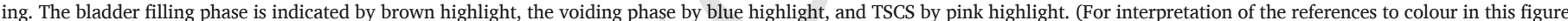
legend, the reader is referred to the web version of this article.)

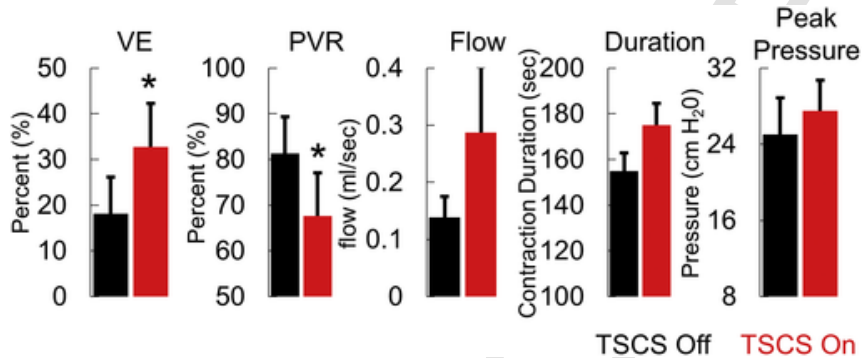

Fig. 4. Mean \pm SE ( $n=5$ subjects), voiding efficiency (VE), post voidal residual (PVR), Flow, Contraction duration (Duration) and peak pressure for the UDS recordings with TSCS Off and On. VE was calculated as voided volume/infused volume, and the PVR was measured by withdrawing the remaining volume from the bladder after completion of voiding. The flow rate was calculated as the voided volume/contraction duration. *Significantly different from TSCS Off at $P<.05$.

lar underlying mechanism for these long latency responses, i.e. activation of spinal micturition centers. The continuous TSCS over $60 \mathrm{~s}$ and partial bladder filling resulted in an overall increase in vesicular pressure above the threshold to initiate voiding. This finding represents a key feature of TSCS-induced voiding, wherein the TSCS activates the neural networks that control LUT function to initiate voiding.

TSCS has recently shown promise for modulation of coordinated stepping in human subjects of both control groups and after an SCI (Gerasimenko et al., 2015a; Gerasimenko et al., 2015b). Recent human studies provide additional support for the activation of common neural structures during epidural spinal cord stimulation and TSCS (Gad et al., 2018b; Gad et al., 2018c). We have also demonstrated the poten- tial utility of TSCS to activate the neural circuitries in the spinal cord of non-human primates to activate the bladder detrusor, urethral and anal external sphincters, and pelvic floor musculature with placement of the stimulation electrode over the thoracolumbar spine and the reference electrode over the abdomen (Gad et al., 2018a). In earlier studies, the reference electrode was similarly placed over the abdominal wall for epidural stimulation studies of stepping in cats (Musienko et al., 2012) or over the iliac crests during TSCS to activate motor circuits in humans (Sayenko et al., 2015). Considering the reference electrode placement over the abdomen, it is possible that TSCS may also activate the abdominal muscles directly. In the presence of possible abdominal muscle contractions, an increase in bladder pressure may take place also without an evoked detrusor contraction. In the present study, we detected on occasion muscular contractions in the lower extremities but were unable to visualize any accessory abdominal contractions, in part as each subject was placed in prone position for the TSCS and urodynamic recordings, and no EMG recordings were obtained from the abdominal wall. Although an increase in bladder pressure and voiding took place during the course of active TSCS in some animals, an evoked voiding response did not take place until after the completion of the TSCS in other subjects, suggesting that a direct TSCS activation of abdominal contractions is not a requirement for TSCS-evoked voiding. However, TSCS was similarly used to activate the lower urinary tract in a human study, and it was demonstrated that unique contractions profiles were observed in the vesicular and abdominal pressure probes causing a non-zero detrusor contraction induced by TSCS only and in the absence of any change in abdominal pressures (Gad et al., 2018b). Also, because the active electrode is positioned directly 
over the spine, the stimulation current is expected to primarily activate the neural networks in the spinal cord. This notion was supported by two separate control experiments in the present study. First, a reversal of the polarity of stimulation at the same intensity and site of stimulation resulted in lower amplitude responses in all components of the LUT. Second, an increase in the strength of the stimulus resulted in larger amplitude EMG responses while maintaining their latencies. Regardless, the addition of a rectal balloon-tip catheter to monitor abdominal pressure in rhesus macaques during future TSCS and urodynamic studies will provide direct and valuable information on a possible contribution of abdominal muscle contractions to bladder pressure changes.

TSCS was used to map the segmental location of neural circuitries for the control of detrusor and urethral pressures, external urethral and anal sphincter activation, and pelvic floor contractions. For this purpose, comprehensive cystometrogram and EMG recordings were performed during the application of TSCS. Prior electrophysiological and anatomical tracer studies in rhesus macaques have demonstrated that the preganglionic parasympathetic neurons are primarily located in the S1-S2 segments (Nadelhaft et al., 1983). Whereas, the external sphincter-innervating motor neurons of Onuf's nucleus typically are positioned within the L6-S1 segments (Gross et al., 2017; Roppolo et al., 1985; Ueyama et al., 1985; Vanderhorst et al., 2000), and the somata of pelvic floor-innervating motor neurons, including those with projections to the levator ani muscle group, reside in the S1-2 segments (Vanderhorst et al., 2000). Our functional maps similarly demonstrated a rostro-caudal segmental representation and identification for the most optimal detrusor and urethral pressure responses, external urethral and anal sphincter EMG activation, and pelvic floor EMG activation. In individual animals, the optimal responses for each of these outcome measures were characteristically within 1-2 inter-spinous process levels among the subjects tested.

It may not be surprising that the relative hot spot locations for LUT functions showed relatively extensive inter-individual variation in the present study, as the vertebral level for the location of the tip of the conus medullaris varied extensively between humans in clinical studies (Saifuddin et al., 1998; Wilson and Prince, 1989). Recent magnetic resonance imaging studies of rhesus macaques demonstrated a similar variation with regards to the rostro-caudal positioning of the sacral spinal cord with the rostral and caudal extent of the conus medullaris at $2.0 \pm 0.3$ and $3.6 \pm 0.4$ vertebral units below the thoracolumbar junction, respectively (Ohlsson et al., 2017). An additional potentially confounding factor for TSCS in rhesus macaques is the presence of a set of supernumerary ribs at the L1 vertebra in about $20 \%$ of subjects (Ohlsson et al., 2017). This anatomical spine variant will likely influence placement of surface electrodes, providing an obvious advantage of the present non-invasive approach compared to a permanently implanted array. Identification of these anatomical and physiological variations should facilitate the procedures for subject-specific optimization of stimulation sites for augmenting voiding function.

The present study was well tolerated by the subjects and shows feasibility of using TSCS to activate neural networks in the lower thoracic and in the lumbosacral spinal cord of nonhuman primates, a novel and non-invasive strategy to augment LUT function. Guided by preceding functional mapping, TSCS in rhesus macaques enabled the activation of micturition reflexes and voiding with improved VE and decreased PVR while maintaining bladder pressures and urine flow within the physiological range.

Our approach offers several advantages that may significantly impact the lives of individuals with impaired bladder function. First, TSCS is non-invasive. If the intervention is not tolerated by the subject, it can be immediately discontinued. Second, TSCS appears to act by neuromodulation of native circuitries and networks devoted to micturition. Third, this intervention may result in plastic changes to the neural circuitry controlling bladder voiding and sensation. Fourth, TSCS may be safely used across a wide age range, as they were tolerated in rhesus macaques with an age span from 3 to 30 years, corresponding to juvenile, adult, and geriatric animals in a clinically relevant model for aging and translational research studies (Didier et al., 2016; Simmons, 2016). Fifth, TSCS modulates the neural circuitry in the lower thoracic and in the lumbosacral spinal cord to augment LUT function in primates within a single session. Sixth, TSCS neuromodulates the spinal circuitry to improve voiding parameters within a single session suggesting that it can be used on a short term basis for neurological disorders that may result in voiding dysfunction. Guided by preceding functional mapping, TSCS enabled the activation of micturition reflexes and voiding with improved VE and decreased PVR while maintaining bladder pressures, contraction duration, and urine flow within the physiological range. We propose that TSCS may next be an attractive candidate to be evaluated for enabling voiding in subjects with impaired LUT function. Currently, thousands of individuals with reduced LUT function have little or no effective non-invasive, non-pharmaceutical therapies available. For these individuals even seemingly small advances in motor or sensory function can be sufficient to expand the range of social comfort in managing bladder function. Developing a treatment to improve bladder function, will not only elevate the quality of life of the individuals with the impairment but also significantly change the lives of their caregivers and the immediate family.

\section{Declaration of Competing Interest}

V.R.E holds shareholder interest in NeuroRecovery Technologies and hold certain inventorship rights on intellectual property licensed by The Regents of the University of California to NeuroRecovery Technologies and its subsidiaries. P.G.and V.R.E holds shareholder interest in spineX Inc. and hold certain inventorship rights on intellectual property licensed by The Regents of the University of California to spineX Inc.

\section{Acknowledgements}

The studies were supported by the Dr. Miriam and Sheldon G. Adelson Medical Research Foundation, the National Institutes of Health (NIH) Office of the Director (P51 OD011107), and the NIH National Center for Advancing Translational Science (NCATS) UCLA CTSI Grant (UL1TR001881).

\section{References}

Abud, E.M., Ichiyama, R.M., Havton, L.A., Chang, H.H., 2015. Spinal stimulation of the upper lumbar spinal cord modulates urethral sphincter activity in rats after spinal cord injury. Am. J. Physiol. Ren. Physiol. 308, F1032-F1040.

Bartley, J.M., Blum, E.S., Sirls, L.T., Peters, K.M., 2013. Understanding clinic options for overactive bladder. Curr. Urol. Rep. 14, 541-548.

Brindley, G.S., Polkey, C.E., Rushton, D.N., 1982. Sacral anterior root stimulators for bladder control in paraplegia. Paraplegia 20, 365-381.

Chang, H.H., Havton, L.A., 2016. A ventral root avulsion injury model for neurogenic underactive bladder studies. Exp. Neurol. 285 (Pt B), 190-196.

Chang, H.H., Yeh, J.C., Ichiyama, R.M., Rodriguez, L.V., Havton, L.A., 2018. Mapping and neuromodulation of lower urinary tract function using spinal cord stimulation in female rats. Exp. Neurol. 305, 26-32.

Didier, E.S., MacLean, A.G., Mohan, M., Didier, P.J., Lackner, A.A., Kuroda, M.J., 2016 Contributions of nonhuman primates to research on aging. Vet. Pathol. 53, 277-290.

Gad, P.N., Roy, R.R., Zhong, H., Lu, D.C., Gerasimenko, Y.P., Edgerton, V.R., 2014. Initiation of bladder voiding with epidural stimulation in paralyzed, step trained rats. PLoS One 9 (9), e108184.

Gad, P.N., Salyards, G.W., Garzel, L.M., Christe, K.L., Edgerton, V.R., Havton, L.A., 2017. Generalized convulsive seizures are associated with ketamine anesthesia in a rhesus macaque (Macaca mulatta) undergoing urodynamic studies and transcutaneous spinal cord stimulation. J. Med. Primatol. 46, 359-363.

Gad, P.N., Kokikian, N., Christe, K.L., Edgerton, V.R., Havton, L.A., 2018. Non-invasive neurophysiological mapping of the lower urinary tract in adult and aging rhesus macaques. J. Neurophysiol. 119 (4), 1521-1527.

Gad, P.N., Kreydin, E., Zhong, H., Latack, K., Edgerton, V.R., 2018. Non-invasive neuromodulation of spinal cord restores lower urinary tract function after paralysis. Front Neurosci. 12, 432.

Gad, P., Lee, S., Terrafranca, N., Zhong, H., Turner, A., Gerasimenko, Y., Edgerton, V.R., 
2018. Noninvasive activation of cervical spinal networks after severe paralysis. J. Neurotrauma 35 (18), 2145-2158. https://doi.org/10.1089/neu.2017.5461.

Gerasimenko, Y., Gorodnichev, R., Moshonkina, T., Sayenko, D., Gad, P., Edgerton, V.R. 2015. Transcutaneous electrical spinal-cord stimulation in humans. Ann. Phys. Rehabil. Med. 58, 225-231.

Gerasimenko, Y.P., Lu, D.C., Modaber, M., Zdunowski, S., Gad, P., Sayenko, D.G., Morikawa, E., Haakana, P., Ferguson, A.R., Roy, R.R., Edgerton, V.R., 2015. Noninvasive reactivation of motor descending control after paralysis. J. Neurotrauma 32, 1968-1980.

Gross, C., Ellison, B., Buchman, A.S., Terasawa, E., VanderHorst, V.G., 2017. A novel approach for assigning levels to monkey and human lumbosacral spinal cord based on ventral horn morphology. PLoS One 12 (5), e0177243.

Horst, M., Heutschi, J., van den Brand, R., Andersson, K.E., Gobet, R., Sulser, T., Courtine, G., Eberli, D., 2013. Multisystem neuroprosthetic training improves bladder function after severe spinal cord injury. J. Urol. 189, 747-753.

Hubscher, C.H., Herrity, A.N., Williams, C.S., Montgomery, L.R., Willhite, A.M., Angeli, C.A., Harkema, S.J., 2018. Improvements in bladder, bowel and sexual outcomes following task-specific locomotor training in human spinal cord injury. PLoS One 13 (1), e0190998.

Lordêlo, P., Teles, A., Veiga, M.L., Correia, L.C., Barroso, U., 2010. Transcutaneous electrical nerve stimulation in children with overactive bladder: a randomized clinical trial. J. Urol. 184, 683-689.

Nadelhaft, I., Roppolo, J., Morgan, C., de Groat, W.C., 1983. Parasympathetic preganglionic neurons and visceral primary afferents in monkey sacral spinal cord revealed following application of horseradish peroxidase to pelvic nerve. J. Comp. Neurol. 216, 36-52.

Ohlsson, M., Nieto, J.H., Christe, K.L., Villablanca, J.P., Havton, L.A., 2017. Radiographic and magnetic resonance imaging identification of thoracolumbar spine variants with implications for the positioning of the Conus Medullaris in rhesus macaques. Anat. Rec. 300, 300-308.
Peters, K.M., Carrico, D.J., Perez-Marrero, R.A., Khan, A.U., Wooldridge, L.S., Davis, G.L., Macdiarmid, S.A., 2010. Randomized trial of percutaneous tibial nerve stimulation versus sham efficacy in the treatment of overactive bladder syndrome: results from the SUmiT trial. J. Urol. 183, 1438-1443.

Roppolo, J.R., Nadelhaft, I., de Groat, W.C., 1985. The organization of pudendal motoneurons and primary afferent projections in the spinal cord of the rhesus monkey revealed by horseradish peroxidase. J. Comp. Neurol. 234, 475-488.

Saifuddin, A., Burnett, S.J., White, J., 1998. The variation of position of the conus medullaris in an adult population. A magnetic resonance imaging study. Spine 23, 1452-1456.

Sayenko, D.G., Atkinson, D.A., Dy, C.J., Gurley, K.M., Smith, V.L., Angeli, C., Harkema, S.J., Edgerton, V.R., Gerasimenko, Y.P., 2015. Spinal segment-specific transcutaneous stimulation differentially shapes activation pattern among motor pools in humans. J. Appl. Physiol. 118, 1364-1374.

Simmons, H.A., 2016. Age-associated pathology in rhesus macaques (Macaca mulatta). Vet. Pathol. 53, 399-416.

Staskin, D.R., Peters, K.M., MacDiarmid, S., Shore, N., de Groat, W.C., 2012. Percutaneous tibial nerve stimulation: a clinically and cost effective addition to the overactive bladder algorithm of care. Curr. Urol. Rep. 13, 327-334.

Ueyama, T., Mizuno, N., Takahashi, O., Nomura, S., Arakawa, H., Matsushima, R., 1985 Central distribution of efferent and afferent components of the pudendal nerve in macaque monkeys. J. Comp. Neurol. 232, 548-556.

Vanderhorst, V.G., Terasawa, E., Ralston, H.J., Holstege, G., 2000. Monosynaptic projec tions from the nucleus retroambiguus to motoneurons supplying the abdominal wall, axial, hindlimb, and pelvic floor muscles in the female rhesus monkey. J. Comp. Neurol. 424, 233-250.

Wilson, D.A., Prince, J.R., 1989. John Caffey award. MR imaging determination of the location of the normal conus medullaris throughout childhood. Am. J. Roentgenol. 152, 1029-1032. 